\title{
Visible Light Induced Photoreductive Dehalogenation Using Carbon Doped ZnS Nanocrystallite
}

\author{
ATUL V. WANKHADE ${ }^{*}$, M. G. DHONDE ${ }^{2}$, N. T. KHATI ${ }^{3}$, \\ SANJAY R. THAKARE ${ }^{4}$ and G. S. GAIKWAD ${ }^{5}$ \\ ${ }^{1}$ Department of Chemistry, Visvesvaraya National Institute of Technology (VNIT), \\ Nagpur, India \\ ${ }^{2}$ Department of Chemistry, Shri Mathuradas Mohota College of Science, Nagpur, India \\ ${ }^{3}$ Department of Chemistry, Priyadarshini College of Engineering, Nagpur, India \\ ${ }^{4}$ Department of Chemistry, Shri Shivaji Science College, Nagpur, India \\ ${ }^{5}$ Department of Chemistry, J.L. Chaturvedi College of Engineering, Nagpur, India \\ atulwa2006@yahoo.co.in
}

Received 23 April 2013 / Accepted 7 June 2013

\begin{abstract}
A visible light activity was induced in $\mathrm{ZnS}$ nanocrystallites through carbon doping using environment friendly biodegradable starch as stabilizer for controlling particle size and as a source for carbon doping. Synthesized materials exhibits higher photocatalytic activity as compared to undoped bulk $\mathrm{ZnS}$ for the reductive dechlorination of chlorinated benzene derivatives to benzene in the presence of isopropyl alcohol as a sacrificial electron donor under visible light irradiation $(\lambda \geq 400$ $\mathrm{nm})$. The structure, morphology, chemical composition and photocatalytic activity of the nano particals have been investigated by XRD, TEM, EDAX and UV-Visible spectrophotometer. Nano size of synthesized C-doped $\mathrm{ZnS}$ has been confirmed by peak broadning in XRD and TEM, while visible light absorption is confirmed by UV-DRS.
\end{abstract}

Keywords: Wurtzite ZnS, Nanocrystallite, Visible light, Photocatalyst, Dehalogenation.

\section{Introduction}

Polyhalogenated aromatic compounds have emerged out as potent environmental noxious waste, because of their high chemical stability and non-biodegradability. Recently advance oxidation processes (AOP) have been a widely used technique for transforming pollutants into harmless substances. Photocatalysis using $\mathrm{TiO}_{2}$ in aqueous medium has been extensively explored for degradation of polyhalogenated pollutants ${ }^{1,2}$. Although it is one of the most economic and promising technique for industrial effluent treatment containing organic pollutants, this photo-oxidation involves formation of free hydroxyl radicals $\left(\mathrm{OH}^{*}\right)$, which in case of polychlorinated compounds leads to the formation of unavoidable and unknown byproducts ${ }^{2,3}$. Further oxidative photodegradation of polychlorinated compound is kinetically unfavored, as these compounds are electron deficient and resist the electrophilic attack of hydroxy radical ${ }^{4}$. 
The toxicity of halogenated organic contaminants decreases with decrease in number of halogen atoms in polyhalogenated compounds ${ }^{5-7}$, therefore, photoreductive dehalogenation approach using semiconductor catalyst like $\mathrm{CdS}$ and $\mathrm{ZnS}$ has been adopted for detoxification of these compounds. Although CdS is capable of absorbing visible light but it exhibits lower photocatalytic activity for reductive dehalogenation ${ }^{8}$. This is because of its less negative conduction band energy (-2.21 V vs. SCE) as compared to nano-sized ZnS (-2.3 V vs. SCE). $\mathrm{ZnS}$ because of its unique photocatalytic properties has been intensively studied ${ }^{9,10}$. Report reveals that, $\mathrm{ZnS}$ nanoparticles exhibits high photocatalytic activity for photoreduction of $\mathrm{CO}_{2}{ }^{11}$ and photocatalytic water splitting for producing $\mathrm{H}_{2}{ }^{12}$.

Wada et al. has reported selective and stepwise photoreductive dehalogenation of chlorinated benzene derivatives using $\mathrm{ZnS}$ nanocrystallites stabilized in $N, N$-dimethylformamide (DMF) under UV-irradiation, where toxic triethylamine was used as sacrificial electron donor $^{13,14}$.

Hongchao Ma et al. reports the synthesis of visible light responsive $\mathrm{ZnO}-\mathrm{ZnS}$ composite supported on activated carbon ${ }^{15}$. Cho et al. has doped carbon in $\mathrm{ZnO}$ nano structure for inducing visible light photocatalytic activity and to avoid the use of hardcore UV light ${ }^{16}$. Inspired by this and in continuation with our earlier efforts ${ }^{17,18}$, here we report synthesis and characterization of carbon doped $\mathrm{ZnS}$ nanoparticles (C-ZnS nanocrystallites) which are capable of catalyzing dechlorination of 1,4-dichloribenzene under the irradiation of visible light using non-hazardous 2-propanol (2-PrOH) as electron donor.

\section{Experimental}

All the chemicals were of analytical grade and were used without any further purification; solutions were prepared using deionised water. Synthesis of C-ZnS nanocrystallite is reported here by the reaction of $\mathrm{Zn}\left(\mathrm{CH}_{3} \mathrm{COO}\right)_{2}$ and $\mathrm{Na}_{2} \mathrm{~S}$ in aqueous medium, using starch solution as stabilizer and precursor for carbon doping. The details of synthesis is as follows, $1.8347 \mathrm{~g}$ $(10 \mathrm{mmol})$ of $\mathrm{Zn}\left(\mathrm{CH}_{3} \mathrm{COO}\right)_{2}$ was dissolved in $2000 \mathrm{~mL}$ of $1 \%$ starch solution under vigorous stirring for 30 minutes. Separately $0.7805 \mathrm{~g}(10 \mathrm{mmol})$ of anhydrous Sodium Sulfide was dissolved in $100 \mathrm{~mL}$ of water and added to the stirring solution of $\mathrm{Zn}\left(\mathrm{CH}_{3} \mathrm{COO}\right)_{2}$ at the rate of $5 \mathrm{~mL} / \mathrm{min}$. The white precipitate obtained was washed thrice with deionized water followed by washing with ethanol and was air dried. Resultant off-white powder was divided into 3 equal parts which were calcined in air at 100,200 and $300{ }^{\circ} \mathrm{C}$ and the corresponding $\mathrm{C}-\mathrm{ZnS}$ nanocrystallite were denoted as $\mathrm{C}-\mathrm{ZnS} 100, \mathrm{C}-\mathrm{ZnS} 200$ and C-ZnS 300 respectively.

\section{Characterizations of carbon doped ZnS nano-crystallites}

$\mathrm{X}$-Ray diffraction (XRD) patterns were obtained using $\mathrm{Cu} \alpha \mathrm{K}$ radiation and were used to determine the phase structures of the samples. The UV-Visible diffuse reflectance spectra were obtained on a UV- $2450 \mathrm{UV}$-visible spectrophotometer (Shimadzu, Japan), using $\mathrm{BaSO}_{4}$ as a reflectance standard. The morphologies of different C-ZnS nanocrystallite were observed using scanning electron microscopy (SEM) and transmission electron microscopy (TEM). Specific surface area and pore volume (textural properties) of prepared catalysts was determined at $-196{ }^{\circ} \mathrm{C}$ from nitrogen adsorption experiment conducted on Micromeritics instrument ASAP $2420 \mathrm{~V} 2.05$ (V2.05 J). All samples were duly degassed overnight at $200{ }^{\circ} \mathrm{C}$ under vacuum prior to its surface characterization. The Brunauer-Emmett-Teller (BET) model was applied to fit $\mathrm{N}_{2}$ adsorption isotherm and evaluate specific surface area $\left(\mathrm{A}_{\mathrm{BET}}\right)$ of prepared catalysts (below $\mathrm{P} / \mathrm{Po}=0.2501$ ). The $t$-plot method allows obtaining values of external surface area $\left(\mathrm{A}_{\mathrm{Ext}}\right)$ namely surface area associated to the non-microporous structure (macroporous and mesoporous). Micropore volume ( $\left.\mathrm{V}_{\text {Micro }}\right)$ was determined at relative 
pressure of 0.995 and $\mathrm{BJH}$ method was applied to the $\mathrm{N}_{2}$ adsorption data to obtain the micropore and mesopore size distribution. The total pore volume $\left(\mathrm{V}_{\text {tot }}\right)$ was also calculated by the adsorption data at $\mathrm{P} / \mathrm{P}_{0}=0.995$. Presence of carbon was estimated by LECO - USA made Carbon - Sulfur analyzer.

\section{Visible-light photocatalytic activity test}

The photocatalytic activity of the prepared catalysts were studied by UV-Visible spectrophotometer (UV-2450, Shimadzu, Japan) in terms of the photocatalytic degradation of $10^{-2} \mathrm{mmol}$ aqueous solution $(\mathrm{pH}=7)$ of 1,4 -dichlorobenzene at ambient temperature. Mixture of $100 \mathrm{~mL}$ of this solution and $7.65 \mathrm{~mL}(0.1 \mathrm{~mole})$ of 2-propanol in borosilicate reactor was irradiated by $100 \mathrm{~W}$ Tungsten filament lamp, placed $10 \mathrm{~cm}$ below the reactor. The photoreactor was cooled by continuous flow of aqueous $\mathrm{NaNO}_{2}$ solution between the lamp and reactor, which also acts as filter for UV light. Optimized dose of $200 \mathrm{mg}$ of photocatalyst was used. The solution was magnetically stirred in dark for 60 min to ensure the establishment of an adsorption-desorption equilibrium between the photocatalyst, 1,4-dichlorobenzene and water before visible light irradiation. The suspension was magnetically stirred and irradiation with visible light, $2 \mathrm{~mL}$ aliquots were then taken out regularly and centrifuged to remove suspended catalyst. The reaction mixture was also analyzed by gas chromatography (GC) with a fused silica column (Shimadzu) using TIC detector.

\section{Results and Discussion}

\section{XRD pattern}

The crystalline nature of the synthesized C-ZnS photocatalyst calcined at different temperature was studied by powder X-ray diffraction (XRD) as shown in Figure 1. The diffraction patterns matches well with the diffraction pattern of ZnS (JCPDS card no. 89-2739) which can be readily indexed as hexagonal wurtzite type $\mathrm{ZnS}$. The (002) peak intensity increased over that of a (100) peak which belongs to wurtzite- $6 \mathrm{H}$, with the lattice parameters of $\mathrm{a}=3.81 \AA$ and $\mathrm{c}=$ $18.69 \AA$ [S. G. P63mc (186)]. The highest intensity of the (002) peak suggests that C- ZnS nano particles are growing along the (002) plane, providing active surface area for photocatalysis. The XRD patterns of the mesoporous C-ZnS nano particles show that the (100), (002) and (101) peaks are overlapping. The broadening of XRD peaks clearly indicates the nanocrystalline nature of $\mathrm{C}-\mathrm{ZnS}$. There was no peak corresponding to carbon indicating that, the carbon may be present as a dopant in the interstitial crystallite of $\mathrm{ZnS}$. The presence of carbon does not alter the wurtzite crystal geometry of $\mathrm{ZnS}$.

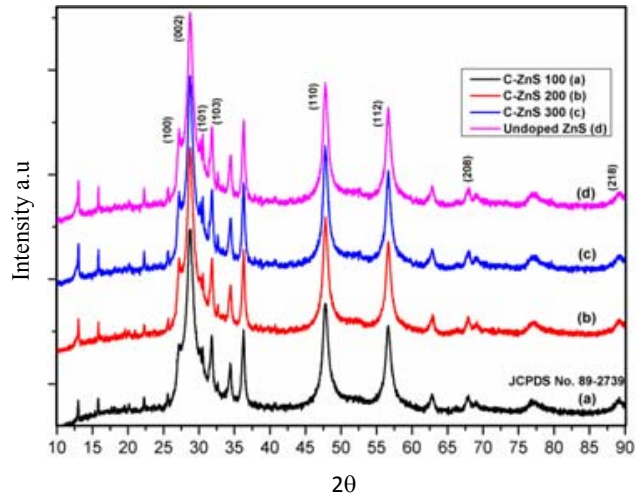

Figure 1. X-ray powder diffraction patterns of $\mathrm{C}-\mathrm{ZnS}$ calcined at different temperature 


\section{UV-Visible diffuse reflectance spectra}

UV-Visible diffuse reflectance spectra of $\mathrm{C}-\mathrm{ZnS}$ calcined at different temperatures are shown in Figure 2. The diffuse reflectance spectra (DRS) of C-ZnS 100, C-ZnS 200 and $\mathrm{C}-\mathrm{ZnS} 300$ show absorbance onset on 425, 420 and $405 \mathrm{~nm}$ respectively, while the absorption range of pure bulk $\mathrm{ZnS}$ is between 200 to $330 \mathrm{~nm}^{11}$. Thus it is observed that $\mathrm{C}-\mathrm{ZnS} 100, \mathrm{C}-\mathrm{ZnS} 200$ and C-ZnS 300 absorbs more light in the range of 400 to $450 \mathrm{~nm}$, as compared to bulk $\mathrm{ZnS}$. The band gap of the synthesized material was calculated by plotting $\log (1 / \mathrm{R})$ verses wavelength. The band gaps optically obtained were approximately 2.917 , 2.952 and $3.06 \mathrm{eV}$ for carbon doped $\mathrm{ZnS}$ calcined at 100,200 and $300{ }^{\circ} \mathrm{C}$ respectively. It is observed that carbon content in prepared material decreases with increase in calcination temperature, thereby increasing the band gap as shown in Table 1 .

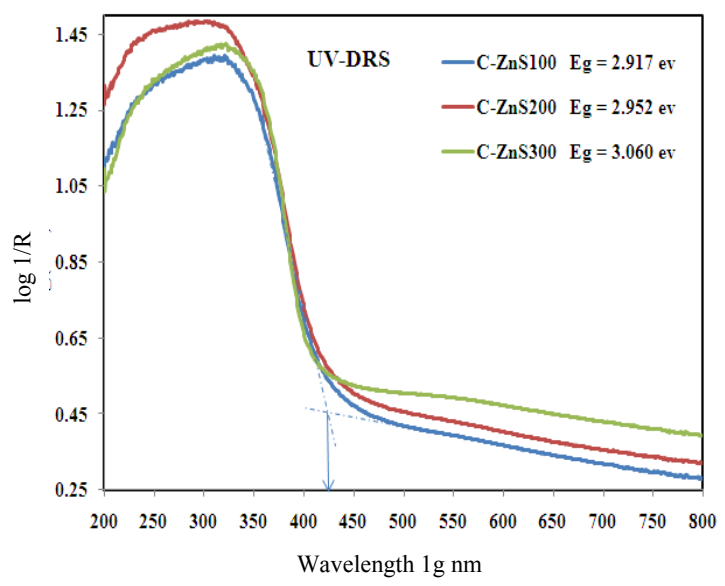

Figure 2. UV-DRS of C-ZnS calcined at different temperature.

Table 1. Carbon estimated by LECO - USA made Carbon - Sulfur analyzer. As Calcinations temperature increases $\mathrm{C} \%$ decreases resulting increase in band gap

\begin{tabular}{ccc}
\hline Sample & Wt \% of carbon in ZnS after calcination & Band Gap eV \\
\hline C-ZnS 100 & 0.22 & 2.917 \\
C-ZnS 200 & 0.18 & 2.952 \\
C-ZnS 300 & 0.10 & 3.060 \\
\hline
\end{tabular}

\section{SEM and TEM analysis}

The morphology and presence of carbon in C-ZnS was studied from the SEM and EDAX Figure $3 \mathrm{a}, 3 \mathrm{~b}$ and $3 \mathrm{c}$. All samples presents strong agglomeration in SEM, however the morphology and particle size of the synthesized samples cannot be resolved by this technique. EDAX pattern of all three C-ZnS shows an intense peak around $1 \mathrm{kev}$ for $\mathrm{Zn}$ and low intensity peak for carbon, this observation along with the XRD pattern gave the convincing evidence for the presence of carbon as dopant in the interstitial crystallite of $\mathrm{ZnS}$. The nanoporous pseudospherical structures of C-ZnS are observed from TEM images. (Figure $4 \mathrm{a}, 4 \mathrm{~b}$ and $4 \mathrm{c}$ ) The particle size distribution obtained from the analysis of TEM images of C-ZnS 200 shows uniform distribution in the range of $15-20 \mathrm{~nm}$, as shown in Figure 5. 


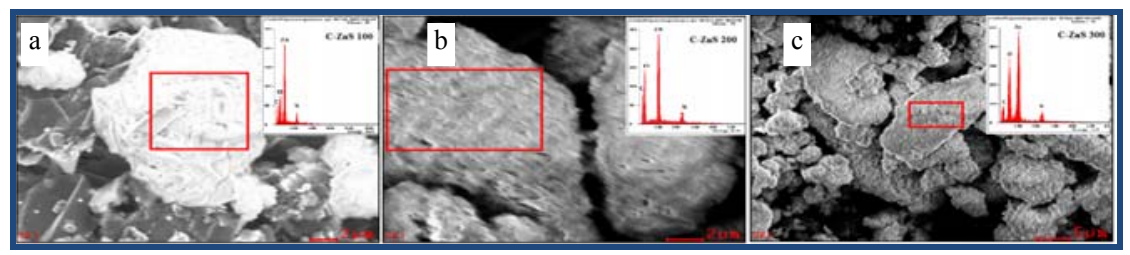

Figure 3. SEM and EDAX of C-ZnS calcined at different temperature

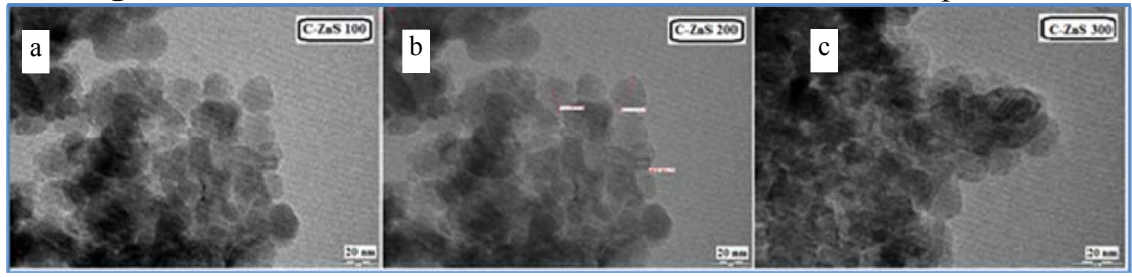

Figure 4. TEM of C-ZnS calcined at different temperature

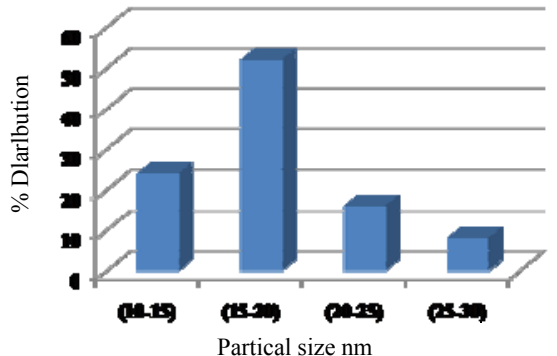

Figure 5. Particle size distribution of C-ZnS 200.

Further TEM image and UV-DRS confirms that the starch used in the preparative strategies has acted as a stabilizer by limiting the particle size below $30 \mathrm{~nm}$ and obviously as a source for carbon doping, which enhanced the absorbance in visible range.

\section{Nitrogen adsorption}

Figure 6 shows the $\mathrm{N}_{2}$ adsorption isotherms at $-196{ }^{\circ} \mathrm{C}$ of catalysts prepared at different temperatures. According to the IUPAC classification, all isotherms exhibited features of type III showing significant increase in the adsorption at low $\mathrm{P} / \mathrm{Po}$ values $(<0.9)$, a broad knee and long plateau up to $\mathrm{P} / \mathrm{Po} \approx 1.0$. Type III is a characteristic of adsorption on macroporous adsorbent with weak adsorbate-adsorbent interactions, pointing towards less porous network form with very small porosity and large external surface area. Table 2 reports various surface textural parameters calculated from $\mathrm{N}_{2}$ adsorption isotherm at $-196{ }^{\circ} \mathrm{C}$. It is observed that all the catalysts showed very high external surface area with comparatively lesser micropore area and volume, where as the BET surface area and external surface area decreases continuously with increasing temperature.

\section{Photocatalytic activity test}

Photocatalytic activity test was investigated by the degradation of aqueous solution of 1,4-dichlorobenzene under visible light irradiation. 1,4-dichlorobenzene showed the maximum absorption at $220 \mathrm{~nm}$. During photodegradation process this absorption peak gradually diminish as shown in Figure 7a. The reaction mixture was also analyzed by gas chromatography (GC) using TIC detector. Figure 7b shows that the peak for 1,4-dichlorobenzene (Retention 
Time 21.027) gradually disappears with increasing irradiation time. The plot of amount \% degradation $\left[\left(\mathrm{C}_{t} / \mathrm{C}_{0}\right) \times 100\right]$ vs. time (min.) for the photodegradation of 1,4-dichlorobenzene is shown in Figure 8.

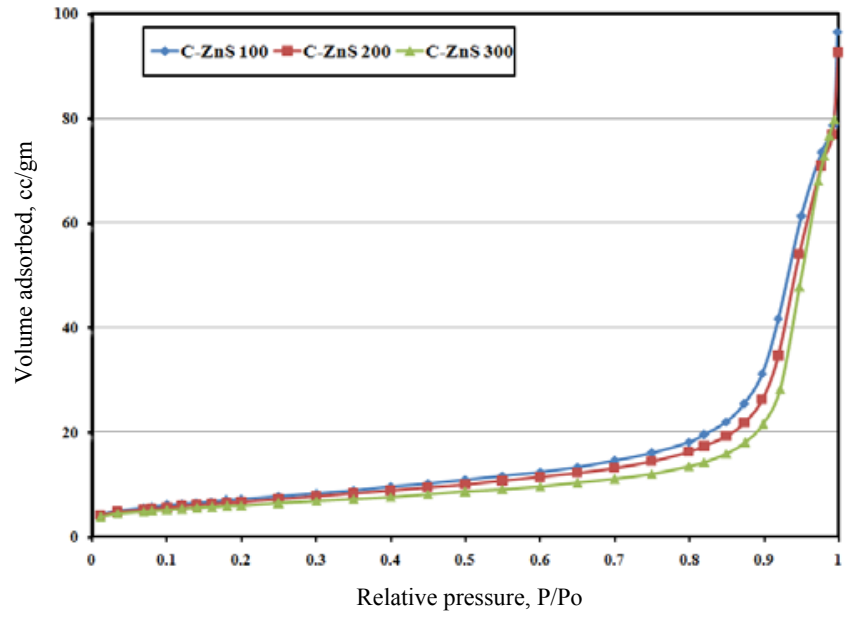

Figure 6. Nitrogen adsorption isotherms of synthesised C-ZnS $-196{ }^{\circ} \mathrm{C}$

Table 2. Physicochemical properties for $\mathrm{C}-\mathrm{ZnS}$ nanocrystallites calcined at various temperatures

\begin{tabular}{lccccccc}
\hline Sample ID & $\begin{array}{c}\mathrm{A}_{\text {Singlept }} \\
\mathrm{m}^{2} / \mathrm{g}\end{array}$ & $\begin{array}{c}\mathrm{A}_{\mathrm{BET}} \\
\mathrm{m}^{2} / \mathrm{g}\end{array}$ & $\begin{array}{c}\mathrm{A}_{\text {Micro }} \\
\mathrm{m}^{2} / \mathrm{g}\end{array}$ & $\begin{array}{c}\mathrm{A}_{\text {Ext }} \\
\mathrm{m}^{2} / \mathrm{g}\end{array}$ & $\begin{array}{c}\mathrm{V}_{\text {Micro }} \\
\mathrm{cm}^{3} / \mathrm{g}\end{array}$ & $\begin{array}{c}\mathrm{V}_{\text {Total }} \\
\mathrm{cm}^{3} / \mathrm{g}\end{array}$ & $\begin{array}{c}\text { Average } \\
\text { pore }\end{array}$ \\
\hline C-ZnS 100 & 25.619 & 26.623 & $*$ & 27.120 & -0.00059 & 0.1214 & 17.615 \\
C-ZnS 200 & 23.657 & 24.343 & 0.8591 & 23.204 & 0.00015 & 0.1439 & 21.166 \\
C-ZnS 300 & 21.071 & 21.487 & 2.6758 & 18.811 & 0.00114 & 0.1239 & 22.411 \\
\hline
\end{tabular}

${ }^{*}$ The micropore area is not reported because the micropore volume is negative. Asinglept Single point surface area, $A_{B E T}$ : Specific surface area obtained by BET equation, $A_{\text {Micro, }} A_{E x t}: t$-Plot Micropore area, $t$-Plot External surface area. $V_{\text {Mic }}, V_{\text {Total }}$ t $t$-Plot Micropore volume, Total pore volume

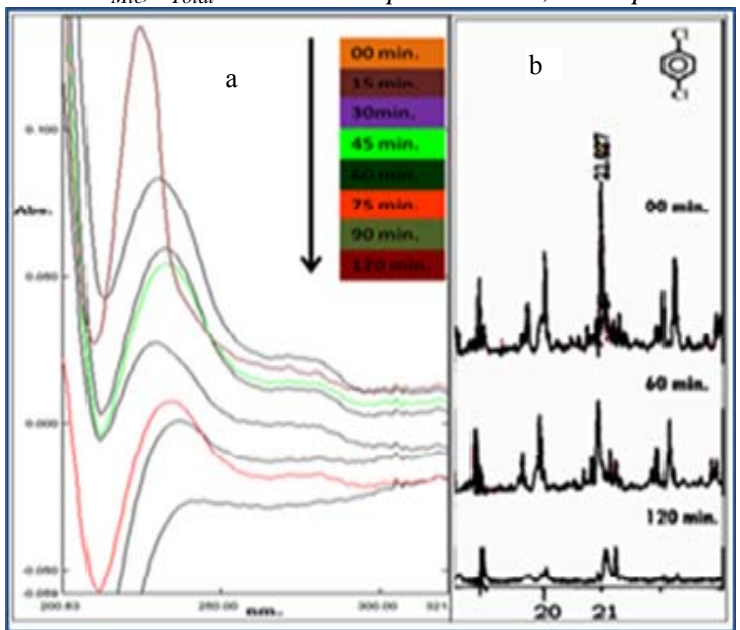

Figure 7. Degradation 1,4-dichlorobenzence with irradiation time a) by UV-Visible spectrophotometer b) by GC 


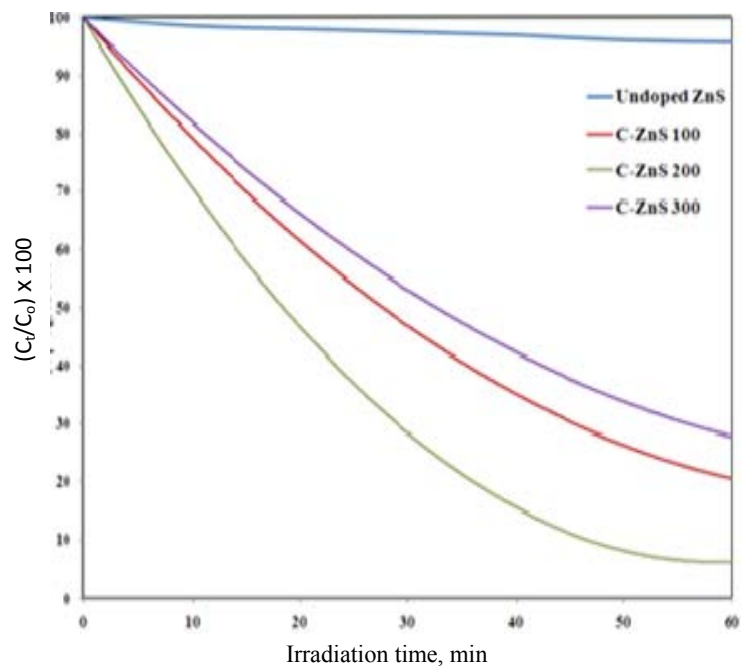

Figure 8. Percentage of 1,4-dichlorobenzence left $\left[\left(\mathrm{C}_{\mathrm{t}} / \mathrm{C}_{0}\right) \times 100\right]$ with irradiation time using $\mathrm{C}$-ZnS nanocrystallites (Dose $200 \mathrm{mg} / 100 \mathrm{~mL}$ ) under visible light irradiation

As reported earlier, $\mathrm{ZnS}$ nanocrystallites in DMF carries out two-electron reduction of $\mathrm{CO}_{2}$ to $\mathrm{HCOOH}^{11}$. Similar to this photogenerated electrons on $\mathrm{C}-\mathrm{ZnS}$ possessing higher reducing power than 1,4-dichlorobenzene (-2.2 V vs. SCE) gives radical anion of 1,4-dichlorobenzene. This radical anion readily undergo dechlorination to generate radicals, which may undergo further reduction, this successive two-electron reduction followed by protonation, causes the stepwise dechlorination of 1,4-dichlorobenzene to give chlorobenzene followed by benzene.

Figure 9 indicates mechanism of photocatalytic dechlorination for prepared C-ZnS catalysts. The electrons responsible for reduction can be attributed to photo oxidation of 2-propanol at valence band of $\mathrm{C}-\mathrm{ZnS}$, resulting in the formation of $2-\mathrm{PrOH}^{+}$. This $2-\mathrm{PrOH}^{+\cdot}$ on further deprotonation giving acetone, as confirmed by peak at Retention Time $16.36 \mathrm{~min}$ in the GC-MS Figure 10.

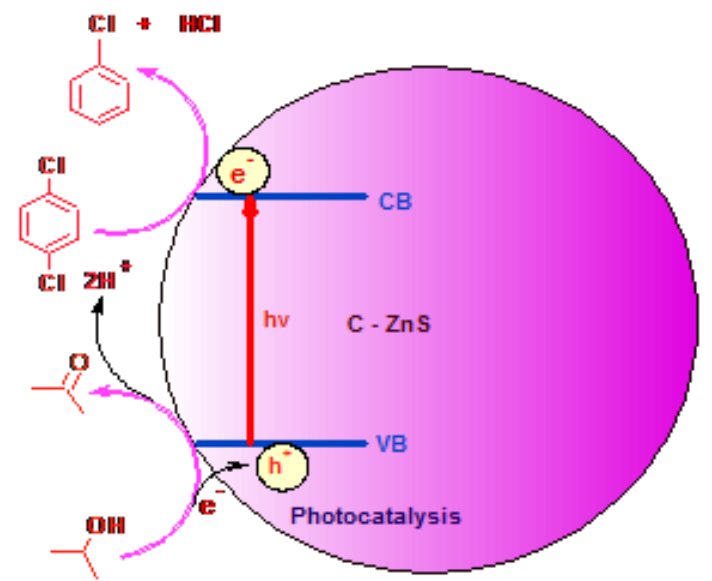

Figure 9. Mechanism of photocatalytic dechlorination for prepared C-ZnS catalysts 

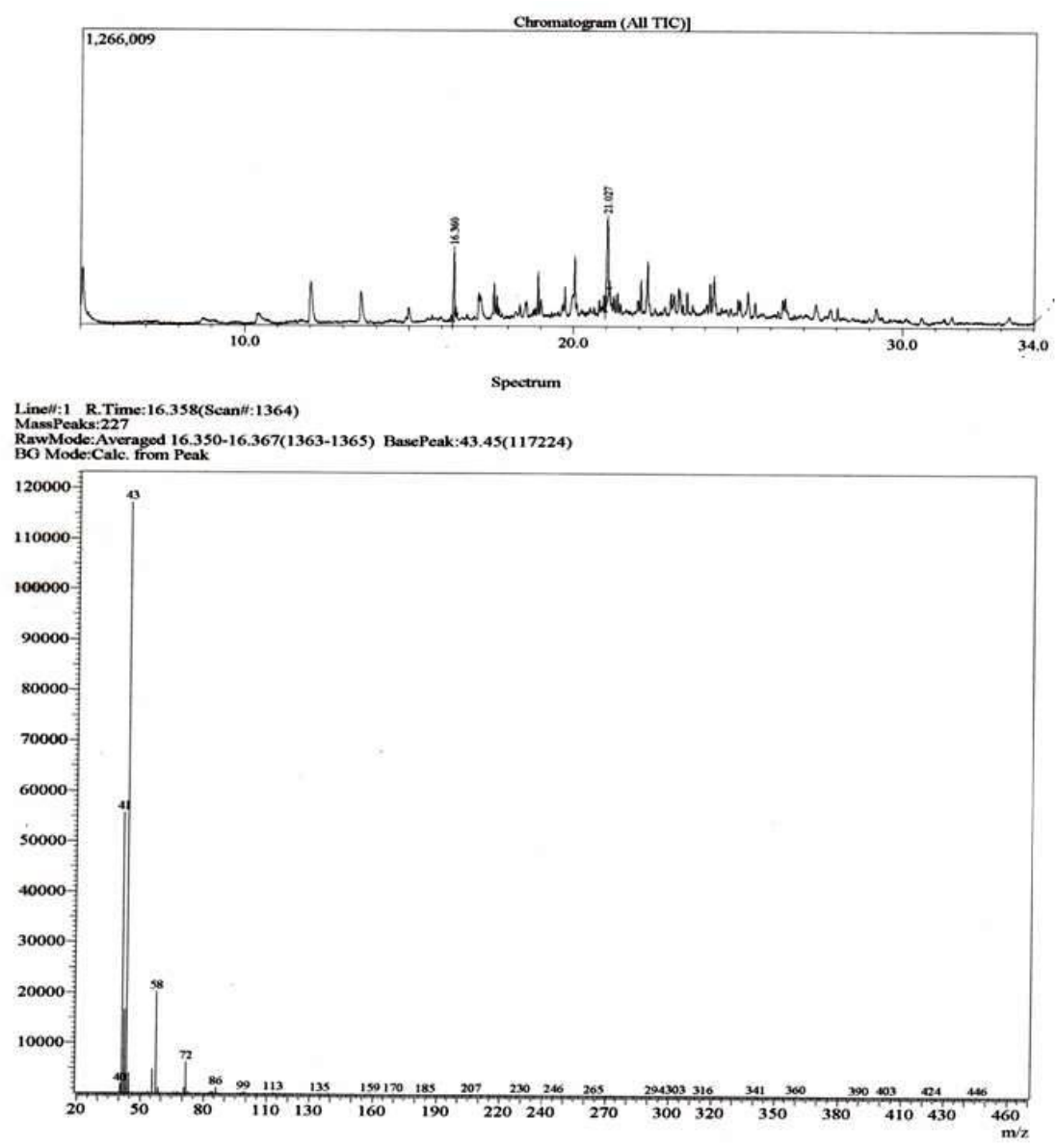

\section{Conclusion}

Figure 10. GC-Mass spectra

We have developed highly stable C-ZnS nanocrystallite by using benign and naturally abundant starch as stabilizer. This visible light active catalyst showed excellent activity for dechlorination of 1,4-dichloro benzene, crucially the entire process was carried out in aqueous medium without using any organic solvent in the reaction as well as during the workup. This photoreduction should provide a new strategy for detoxification of hazardous chlorinated aromatic compounds under minimum-energy conditions.

\section{References}

1. Pandiyan T, Martinez R, Martinez J, Amezcua G and Martinez-Carrillo M, $J$ Photochem Photobiol A Chem., 2002, 146(3), 149-155;

DOI:10.1016/S1010-6030(01)00606-2

2. Theurich J, Lindner M and Bahnemann D W, Langmuir, 1996, 12(26), 6368-6376; DOI:10.1021/la960228t

3. Werner R H and Yao C C D, Environ Sci Technol., 1992, 26(5), 1005-1013; DOI:10.1021/es00029a021

4. Wada Y, Taira M, Zheng D and Yanagida S, New J Chem., 1994, 18, 589-596. 
5. Berry F J, Smart L E, Sai Prasad P S, Lingaiah N and Rao P K, Appl Catal A: General, 2000, 204(2), 191-201; DOI:10.1016/S0926-860X(00)00515-9

6. Aramendia M A, Borau V, Garcia I M, Jimenez C, Lafont F, Marinas A, Marinas J M and Urbano F J, J Catal., 1999, 187(2), 392-399; DOI:10.1006/jcat.1999.2632

7. Yakovlev V A, Terskikh V V, Simagina V I and Likholobov V A, J Mol Catal A, 2000, 153(1-2), 231-236; DOI:10.1016/S1381-1169(99)00352-0

8. Yin H, Wade Y, Kitamura T and Yanagida S, Environ Sci Technol., 2001, 35(1), 227-231; DOI:10.1021/es001114d

9. Chen D, Huang F, Ren G, Li D, Zheng M, Wang Y and Lin Z, Nanoscale, 2010, 2, 2062-2064; DOI:10.1039/C0NR00171F

10. Hu J S, Ren L L, Guo Y G, Liang H P, Cao A M, Wan L J and Bai C L, Angew Chem Int Ed., 2005, 44(6), 1269-1273; DOI:10.1002/anie.200462057

11. Fujiwara H, Hosokawa H, Murakoshi K, Wada Y and Yanagida S, Langmuir, 1998, 14(18), 5154-5159; DOI:10.1021/la9801561

12. Tsuji I, Kato H, Kobayyashi H and Kudo A, J Am Chem Soc., 2004, 126(41), 1340613413; DOI:10.1021/ja048296m

13. Wade Y, Yin H, Kitamura T and Yanagida S, Chem Commun., 1998, 24, 2683-2684; DOI:10.1039/A806035E

14. Shiraishi Y, Takeda Y, Sugano Y, Ichikawa S, Tanakac S and Hirai T, Chem Commun., 2011, 47, 7863-7865; DOI:10.1039/C1CC12087E

15. Hongchao Ma, Han J, Fu Y, Song Y, Yu C and Dong X, Appl Catal B: Environ., 2011, 102(3-4), 417-423; DOI:10.1016/j.apcatb.2010.12.014

16. Cho S, Jang J W, Lee J S and Lee K H, Cryst Eng Comm., 2010, 12, 3929-3935; DOI:10.1039/C0CE00063A

17. Rahal R, Wankhade A, Cha D, Fihri A, Chikh S O, Patil U and Polshettiwar V, RSC Adv., 2012, 2, 7048-7052; DOI:10.1039/C2RA21104A

18. Ingole A R, Thakare $\mathrm{S} \mathrm{R}$, Khati $\mathrm{N} \mathrm{T}$, Wankhade $\mathrm{A} \mathrm{W}$ and Burghate $\mathrm{D} \mathrm{K}$, Chalcogenide Lett., 2010, 7, 485-489. 